\title{
Grado de adherencia a la recomendación \\ de actividad física, sedentarismo y riesgo cardiovascular \\ en adolescentes de enseñanza media de Punta Arenas, Chile
}

\author{
Degree of adherence to physical activity \\ recommendation, sedentary lifestyle and cardiovascular risk \\ in teenagers from Punta Arenas, Chile
}

\begin{abstract}
O bjective: The aim of this study was to identify the degree of compliance to the global recommendations on physical activity and its association with cardiovascular risk, anthropometric and socio-demographic variables in teenagers from the district of Punta Arenas, Chile. Subjects and M ethods: A random, stratified and proportional sample was obtained of 322 students from private, private-subsidized and public local schools. Global physical activity questionnaire (GPAQ v2), and anthropometric measurements of weight, height, waist circumference, and classification of nutritional status were applied according to the standards of the Chilean Ministry of Health. Results: $50.31 \%$ of the sample showed a normal nutritional status, $48.14 \%$ presented overweight status. We also found $666.8 \mathrm{~min} /$ day of average sedentary lifestyle between the means by gender. The female gender presents an $\mathrm{O}$ : 1.9; $95 \%$ IC: 1.16 to 3.11 according to WHO recommendations. Discussion: It was found that most of the students resulted in the category "sufficiently active" as WHO recommended. An $82.30 \%$ showed no high cardiovascular risk. Conclusion: The level of physical activity would not be influenced by nutritional status, showing that the majority of students perform moderate physical activity on their free time.
\end{abstract}

Key words: Physical activity; GPAQ ; cardiovascular risk; teenagers; nutritional status.

\section{INTRODUCCIÓN}

La salud de los adolescentes tiene relación con su propia conducta, la cual se ve influida en gran medida por el ambiente en que vive. La adolescencia después de la niñez, es la segunda etapa más vulnerable del ciclo vital, donde se adquieren la mayoría de los hábitos que tienen consecuencias negativas para la salud a largo plazo, tales como la inactividad física y la malnutrición por exceso, entre otras, constituyéndose en factores de riesgo para enfermedades crónicas no trasmisibles, las que se presentan cada vez con mayor frecuencia a tempranas edades (1).

De los diez factores de riesgo identificados por la OMS como claves para el desarrollo de las enfermedades crónicas, cinco están estrechamente relacionados con el ejercicio físico y la alimentación. Además de la obesidad, se citan el sedentarismo, hipertensión arterial, hipercolesterolemia y consumo insuficiente de frutas y verduras (2).

La inactividad física, constituye el cuarto factor de riesgo más importante de mortalidad en el mundo ( $6 \%$ de defunciones a nivel mundial). Sólo la superan la presión arterial elevada (13\%), el consumo de tabaco (9\%) y la glicemia
Pedro Quintana P. (1)

Paola Aravena M. (1)

Waldo Aranda Ch. (2)

Miriam Díaz M. (3)

Brenda Soto O. (3)

Esteban Muñoz E. (3)

(1) Departamento de Kinesiología, U niversidad de Magallanes, Punta Arenas, Chile. (2) Departamento de Bioestadística. Universidad Diego Portales, Santiago, Chile. (3) Carrera de Kinesiología. Universidad de Magallanes, Punta Arenas, Chile.

Dirigir la correspondencia a: Profesor Pedro Q uintana Peña Avenida Bulnes 01855 Casilla113-D Punta Arenas. Chile

Teléfono: 56-61-2299616

E-mail:pedro.quintana@umag.cl

Este trabajo fue recibido el 16 de Diciembre de 2014 y aceptado para ser publicado el 30 de Septiembre de 2015.

elevada (6\%) (3).

Los resultados de la sistematización de las fichas ClapOMS/OPS a nivel país publicados el año 2012, evidenciaron que los(as) adolescentes realizan 4,3 horas de actividad física (AF) semanales, lo que clasifica a este grupo como sedentarios, definido sedentarismo como la práctica de actividad física menor de una hora al día $(4,5,14)$.

EI SIMCE Nacional de Actividad Física 2012 aplicado a escolares de $8^{\circ}$ básico (6), que evalúa la condición física estructural y funcional, encontró que del total de estudiantes, 56\% tiene un IMC normal, mientras que $44 \%$ presenta sobrepeso u obesidad. Según sexo, $53 \%$ de las mujeres y $60 \%$ de los hombres tiene un IMC normal, mientras que $47 \%$ y $40 \%$, respectivamente, presenta sobrepeso u obesidad. En cuanto a resultados de la medición de perímetro de cintura, esta prueba evidenció que, del total de estudiantes, $23 \%$ presentaría riesgo de desarrollar enfermedades cardíacas y metabólicas en la edad adulta. Según la distribución por sexo, 25\% de las mujeres y $22 \%$ de los hombres presentaría este factor de riesgo. Respecto al porcentaje de estudiantes según los aspectos estructurales de la condición física, del total país $8 \%$ tiene un nivel satis- 
factorio, mientras que en Magallanes $11 \%$ de los estudiantes se encontró en este nivel. Sobre los aspectos funcionales de la condición física del total de estudiantes país, 30\% tiene un nivel satisfactorio, mientras que en la Región de Magallanes, $39 \%$ de los estudiantes estaba en dicho nivel.

La encuesta mundial de salud escolar chilena del 20042005 (EMSE), reveló 85\% de sedentarismo en hombres y $92,8 \%$ en mujeres de 13 a 15 años (5).

JUNAEB, por su parte divulgó que, en estudiantes de primer año de enseñanza media en su informe 2014 en la región de Magallanes y Antártica Chilena existe 2,9\% de riesgo de desnutrición, 48,4\% de prevalencia de estado nutricional normal, y $32,7 \%$ de prevalencia de riesgo de obesidad $(32,4 \%$ en niños y 33,1\% en niñas) (7).

Relacionado con lo anterior, cabe destacar que diversos trabajos epidemiológicos realizados en adultos han evaluado y validado el perímetro de cintura, como un importante predictor, no sólo de factores de riesgo cardiovascular, sino de diabetes tipo 2 y enfermedad coronaria. Esta medición, también ha sido validada en niños como predictor de síndrome metabólico (8-9). Datos reportados en Estados Unidos de Norteamérica (EE.UU.) hablan de $4 \%$ a $9 \%$ de síndrome metabólico en la población general de adolescentes, y 38\% a $50 \%$ en adolescentes obesos (10-11). En Chile, se han reportado cifras similares (12-13).

El objetivo del presente estudio fue identificar el grado de adherencia a las recomendaciones mundiales sobre actividad física, entregando información más detallada y específica en cuanto a tiempo, intensidad y frecuencia de la participación en esta, así como su asociación con el riesgo cardiovascular, va- riables antropométricas y socio-demográficas en adolescentes de la comuna de Punta Arenas. Sustentado lo anterior, por la escasa información en la Región, respecto a la actividad física en este grupo etario.

\section{SUJETOS Y MÉTODOS}

El tipo de estudio fue cuantitativo, analítico y transversal. El universo estaba constituido por 1.975 estudiantes de ambos sexos, basado en los antecedentes otorgados por la Secretaría Regional Ministerial de Educación de Magallanes y de la Antártica Chilena, sobre la matrícula de primer año de Enseñanza Media en todos los establecimientos educacionales de la comuna de Punta Arenas al mes de mayo 2014. De los cuales, 190 estudiantes pertenecían a colegios particulares-pagados, 889 estudiantes a colegios particulares-subvencionados y 896 estudiantes a colegios municipalizados.

Para calcular el tamaño de la muestra se usó un nivel de confianza del 95\%, un margen de error total de un 5\%, para un criterio de máxima variabilidad, obteniéndose una muestra de 322 estudiantes. Mediante una distribución proporcional se distribuyó la muestra en los tres estratos considerados en el estudio, resultando 31 estudiantes de la enseñanza particular pagada, 145 estudiantes de la enseñanza particular subvencionada y 146 estudiantes de la enseñanza Municipal.

Las variables independientes estudiadas fueron sexo y tipo de establecimiento y las variables dependientes, nivel de actividad física, IMC y perímetro de cintura.

La medición de la actividad física (AF) estuvo a cargo de un profesional kinesiólogo y la colaboración de un estudiante de 4to año de la carrera de kinesiología, se utilizó la versión 2

\section{CUESTIONARIO MUNDIAL SOBRE ACTIVIDAD FÍSICA (GPAQ)}

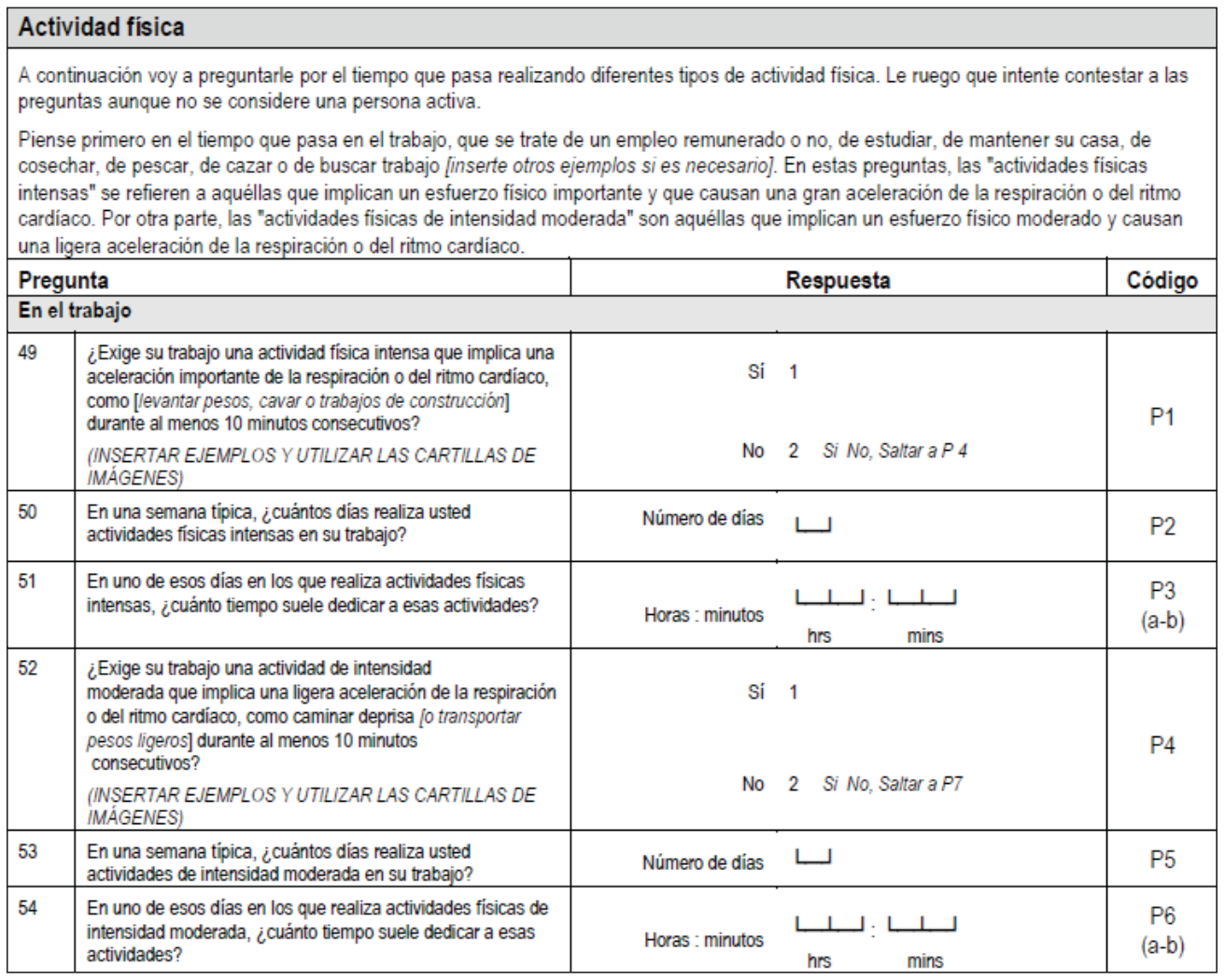




\begin{tabular}{|c|c|c|c|}
\hline \multicolumn{4}{|c|}{ Para desplazarse } \\
\hline \multicolumn{4}{|c|}{$\begin{array}{l}\text { En las siguientes preguntas, dejaremos de lado las actividades fisicas en el trabajo, de las que ya hemos tratado. } \\
\text { Ahora me gustaria saber cómo se desplaza de un sitio a otro. Por ejemplo, cómo va al trabajo, de compras, al mercado, al lugar de culto } \\
\text { [insertar otros ejemplos si es necesario] }\end{array}$} \\
\hline 55 & $\begin{array}{l}\text { ¿Camina usted o usa usted una bicicleta al menos } 10 \text { minutos } \\
\text { consecutivos en sus desplazamientos? }\end{array}$ & $\begin{array}{ll}\text { Si } & 1 \\
\text { No } & 2 \text { Si No, Saltar a } P 10\end{array}$ & P7 \\
\hline 56 & $\begin{array}{l}\text { En una semana tipica, ¿cuántos dias camina o va en bicicleta } \\
\text { al menos } 10 \text { minutos consecutivos en sus desplazamientos? }\end{array}$ & Número de dias $\sqcup$ & P8 \\
\hline 57 & $\begin{array}{l}\text { En un dia típico, ¿cuánto tiempo pasa caminando o yendo en } \\
\text { bicicleta para desplazarse? }\end{array}$ & 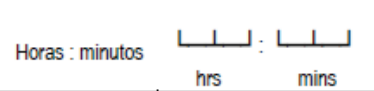 & $\begin{array}{l}\text { P9 } \\
(a-b)\end{array}$ \\
\hline \multicolumn{4}{|c|}{ En el tiempo libre } \\
\hline \multicolumn{4}{|c|}{$\begin{array}{l}\text { Las preguntas que van a continuación excluyen la actividad fisica en el trabajo y para desplazarse, que ya hemos mencionado. Ahora me } \\
\text { gustaria tratar de deportes, fitness u otras actividades fisicas que practica en su tiempo libre [inserte otros ejemplos si llega el caso]. }\end{array}$} \\
\hline 58 & $\begin{array}{l}\text { ¿En su tiempo libre, practica usted deportes/fitness intensos } \\
\text { que implican una aceleración importante de la respiración o } \\
\text { del ritmo cardiaco como [correr, jugar al fútbol] durante al } \\
\text { menos } 10 \text { minutos consecutivos? } \\
\text { (INSERTAR EJEMPLOS Y UTILIZAR LAS CARTILLAS DE } \\
\text { IMÁGENES) }\end{array}$ & No 2 Si No, Saltar a 13 & P10 \\
\hline 59 & $\begin{array}{l}\text { En una semana tipica, ¿cuántos dias practica usted } \\
\text { deportes/fitness intensos en su tiempo libre? }\end{array}$ & Número de dias & P11 \\
\hline 60 & $\begin{array}{l}\text { En uno de esos dias en los que practica deportes/fitness } \\
\text { intensos, ¿cuánto tiempo suele dedicar a esas actividades? }\end{array}$ & 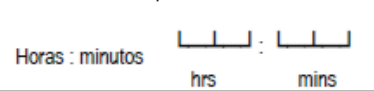 & $\begin{array}{l}\mathrm{P} 12 \\
(\mathrm{a}-\mathrm{b})\end{array}$ \\
\hline
\end{tabular}

\begin{tabular}{|c|c|c|c|}
\hline \multicolumn{4}{|c|}{ SECCIÓN PRINCIPAL: Actividad física (en el tiempo libre) sigue. } \\
\hline \multicolumn{2}{|c|}{ Pregunta } & Respuesta & \multirow{2}{*}{$\begin{array}{l}\text { Código } \\
\text { P13 }\end{array}$} \\
\hline 61 & $\begin{array}{l}\text { ¿En su tiempo libre practica usted alguna actividad de } \\
\text { intensidad moderada que implica una ligera aceleración } \\
\text { de la respiración o del ritmo cardiaco, como caminar } \\
\text { deprisa, [ir en bicicleta, nadar, jugar al volleyball] } \\
\text { durante al menos } 10 \text { minutos consecutivos? } \\
\text { (INSERTAR EJEMPLOS Y UTILIZAR LAS CARTILLAS } \\
\text { DE IMÁGENES) }\end{array}$ & No 2 Si No, Saltar a P16 & \\
\hline 62 & $\begin{array}{l}\text { En una semana tipica, ¿cuántos dias practica usted } \\
\text { actividades fisicas de intensidad moderada en su tiempo } \\
\text { libre? }\end{array}$ & Número de dias & P14 \\
\hline 63 & $\begin{array}{l}\text { En uno de esos dias en los que practica actividades } \\
\text { fisicas de intensidad moderada, ¿cuánto tiempo suele } \\
\text { dedicar a esas actividades? }\end{array}$ & Horas : minutos $\underbrace{\perp \perp ـ ـ}_{\text {hrs }}$ & $\begin{array}{l}\text { P15 } \\
(a-b)\end{array}$ \\
\hline \multicolumn{4}{|c|}{ Comportamiento sedentario } \\
\hline \multicolumn{4}{|c|}{$\begin{array}{l}\text { La siguiente pregunta se refiere al tiempo que suele pasar sentado o recostado en el trabajo, en casa, en los desplazamientos o con sus } \\
\text { amigos. Se incluye el tiempo pasado [ante una mesa de trabajo, sentado con los amigos, viajando en autobús o en tren, jugando a las cartas } \\
\text { o viendo la televisión], pero no se incluye el tiempo pasado durmiendo. } \\
\text { [INSERTAR EJEMPLOS] (UTILIZAR LAS CARTILLAS DE IMÁGENES) }\end{array}$} \\
\hline 64 & $\begin{array}{l}\text { ¿Cuándo tiempo suele pasar sentado o recostado en un } \\
\text { dia típico? }\end{array}$ & Horas : minutos $\underbrace{\perp}_{\mathrm{hrs}} \underbrace{\perp \perp}_{\text {mins }}$ & $\begin{array}{l}\text { P16 } \\
(\mathrm{a}-\mathrm{b})\end{array}$ \\
\hline
\end{tabular}

del cuestionario mundial de actividad física GPAQ (GPAQv2). Este cuestionario está compuesto por 16 preguntas sobre la actividad física efectuada en una semana habitual, diferenciándola en ámbitos de realización. Contiene preguntas que dan información sobre la intensidad de la AF (baja, moderada, alta), la frecuencia (días en una semana habitual) y la duración (horas y minutos en un día habitual) de las actividades físicas desarrolladas en tres dominios: (1) ocupación (incluyendo empleo remunerado o no remunerado, estudios, tareas domésticas o búsqueda de trabajo), (2) desplazamientos (caminando o en bicicleta para ir de un lugar a otro) y (3) tiempo libre (ocio). También contempla una pregunta sobre el comportamiento sedentario (tiempo que se suele pasar sentado o recostado, sin considerar el tiempo que se pasa durmiendo por la noche).

Este cuestionario (GPAQ) ha sido validado y ampliamente utilizado para evaluar los patrones de AF (14).
Para el desarrollo del presente estudio, se utilizó la versión disponible en español del GPAQv2, sin efectuar cambios al contenido original del cuestionario.

Para análisis de los datos se siguieron las indicaciones según protocolo de análisis GPAQ. Calculando en primer término los minutos diarios de AF global para los distintos niveles de intensidad: "baja", "moderada" y "vigorosa", y en cada uno de los ámbitos o dominios: "trabajo", "transporte" y durante el "tiempo libre".

La clasificación de sujetos "suficientemente activos", se realizó según clasificación OMS para niños y jóvenes de 5 a 17 años, quienes deberían acumular un mínimo de 60 minutos diarios de actividad física moderada o vigorosa (16).

Las variables antropométricas estudiadas fueron peso, talla y perímetro de cintura. La determinación del peso $(\mathrm{kg})$ se efectuó mediante una balanza mecánica con tallímetro 
marca ADE con capacidad de $200 \mathrm{~kg}$ y graduación de $100 \mathrm{~g}$, previamente calibrada. La estatura $(\mathrm{m})$ se obtuvo al medir a través del tallímetro incorporado en la balanza mecánica de precisión de $1 \mathrm{~mm}$. El perímetro de cintura se obtuvo con una cintra métrica plástica no deformable.

El estado nutricional se determinó utilizando el IMC (Índice de Masa Corporal) que se calcula según el peso dividido por la talla al cuadrado (IMC= peso/talla2). La clasificación del estado nutricional se realizó según curvas CDC/NCHS 2000 y norma técnica de evaluación nutricional de niños y niñas de 6 a 18 años, considerando bajo peso aquel adolescente que se encontraba bajo el p10; normal entre el p10 y el p85; riesgo de obesidad, entre p85 y el p95 y con obesidad, $\geq$ del p95 (1).

La evaluación de la circunferencia de cintura se utilizó como indicador de presencia de grasa intra abdominal y como factor predictivo de riesgo cardiometabólico, para ello se realizó la medición en el punto medio entre la última costilla y la cresta ilíaca, considerándose aumentada cuando fue mayor o igual al percentil 90 según las tablas de circunferencia de cintura de adolescentes de Nhanes III, Fernández JR (1). Las mediciones estuvieron a cargo de una profesional nutricionista, con la colaboración de dos estudiantes de 4to año de la carrera de kinesiología.

Los criterios de inclusión fueron todos los estudiantes que se encuentren matriculados en primer año de enseñanza media de los diferentes establecimientos educacionales de la comuna de Punta Arenas al mes de Mayo del año 2014. Los criterios de exclusión fueron: no aceptar participar en la investigación (rechazo del Consentimiento Informado), no estar matriculado a Mayo del año 2014 en 1er año educación media, aquellos que presenten algún impedimento para completar el cuestionario o realizar las mediciones antropométricas para evaluación nutricional objetiva, aquellos que no asistan al colegio el día de aplicación de cuestionario y evaluación nutricional, y suspensión académica por motivos extra-programáticos.

El protocolo de investigación fue visado por el Comité de Bioética de la Universidad de Magallanes. Para la aplicación del cuestionario GPAQ, toma de medidas antropométricas, registro y utilización posterior de los datos, se obtuvo previamente la autorización de autoridades educacionales y el consentimiento informado de padres/apoderados/estudiantes (formato OPS) (15)

\section{Análisis estadístico}

Para la tabulación y análisis de los resultados se utilizó Microsoft Office Excel 2010 y en el análisis se usó el programa SPSS versión 21 para realizar el Test Chi2, T de student y análisis de varianza (ANOVA).

Se comenzó analizando los resultados a partir de estadígrafos descriptivos (media, desviación estándar y porcentajes) en las variables IMC, perímetro de cintura y minutos de actividad física al día. Posteriormente, se ordenó la información en base a tablas para organizarlas por sexo, tipo de establecimientos y nivel de actividad física, trasladándolos a gráficos de barras, y así poder visualizar de mejor manera los datos obtenidos. Se analizó el grado de adherencia a las recomendaciones de la OMS, según sexo y tipo de establecimiento, siendo negativo cuando el p>0,05. Asimismo, se realizó el cálculo del OR entre las variables recomendación de actividad física de la OMS y sexo. También, se aplicó ANOVA, entre los minutos de actividad física y sedentarismo al día, con sexo y tipo de establecimiento.

\section{RESULTADOS}

La muestra total compuesta por 175 mujeres (54,3\%), y 147 hombres (45,7\%), cuyas edades fluctuaban entre 14 y 15 años de edad (38,2\% tenían 14 años, y 61,8\% tenían 15 años), según lo esperado para adolescentes que cursan primer año de enseñanza media.

Los establecimientos educacionales se agruparon en tres categorías: establecimientos municipales (45,3\%), particularessubvencionados (45\%) y particulares-pagados $(9,7 \%)$.

De los estudiantes evaluados, 50,31\% tenían un estado nutricional normal, y 48,14\% presentaba malnutrición por exceso. Porcentualmente, existe una mínima diferencia (2,17\%) entre el diagnóstico nutricional normal y estudiantes con malnutrición por exceso, en relación a los niveles de actividad física (gráfico 1).

Se destaca que existía una mayor prevalencia de obesidad en los establecimientos municipalizados presentando un $29,45 \%, 17,93 \%$ en los subvencionados y $12,9 \%$ en los establecimientos particulares (gráfico 2).

Según el tipo de establecimiento y la media observada del IMC, fue: municipales $24,4 \mathrm{~kg} / \mathrm{m} 2$; particulares $24,2 \mathrm{~kg} / \mathrm{m} 2$; subvencionados $23,3 \mathrm{~kg} / \mathrm{m} 2$. Respecto a lo anterior, no existe

\section{GRÁFICO 1}

Diagnóstico nutricional y nivel de actividad física (AF).

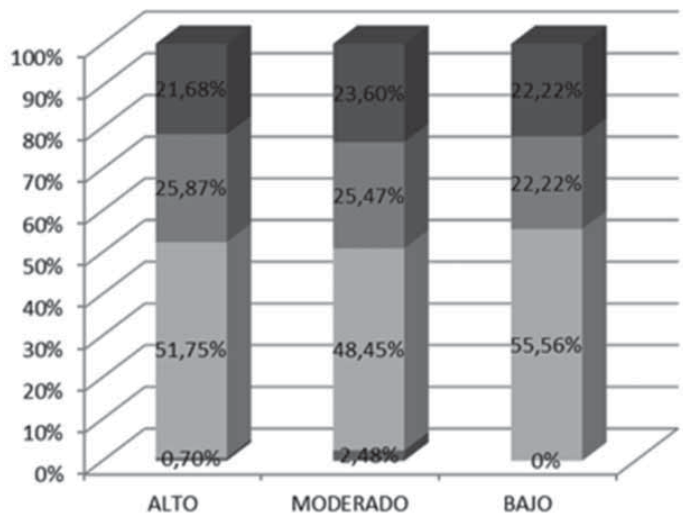

obesidad

E Riesgo de Obesidad

Eeso Normal

- Bajo Peso 
correlación entre estas variables $(p=0,147)$, tampoco existen diferencias significativas entre estas variables $(p=0,239)$.

El $82,3 \%$ del total de la muestra no presentaba riesgo cardiovascular de acuerdo al perímetro de cintura. Porcentualmente, las mujeres presentaban $8,74 \%$ menos riesgo cardiovascular con respecto a los varones. El 17,7\% del total de la muestra presentaba riesgo cardiovascular (tabla 1).

Un 94,4\% del total de la muestra realizaba actividad física de intensidad alto-moderado, donde el 50\% corresponde a actividad física de nivel moderado, y $44,4 \%$ de nivel alto, en la tabla 1 se detalla la distribución por género.

Referente a las recomendaciones de actividad física de la

\section{GRÁFICO 2}

Diagnóstico nutricional y tipo de establecimiento educacional.

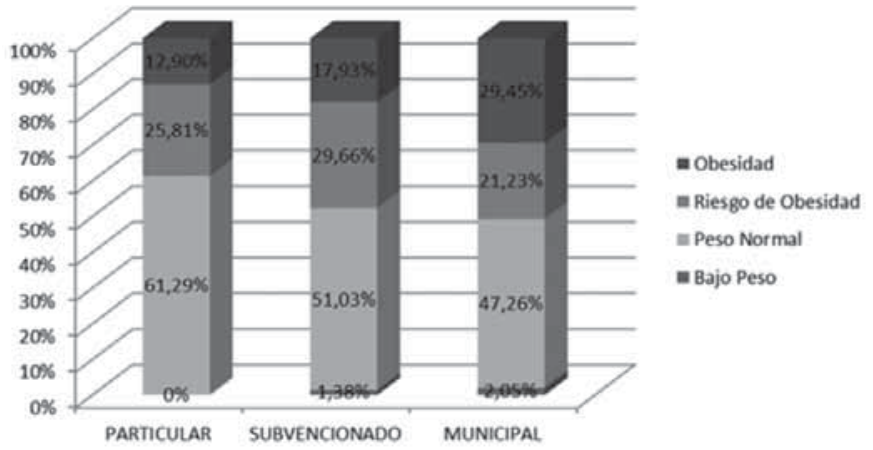

GRÁFICO 3

Media min/día de AF según ámbito y tipo de establecimiento.

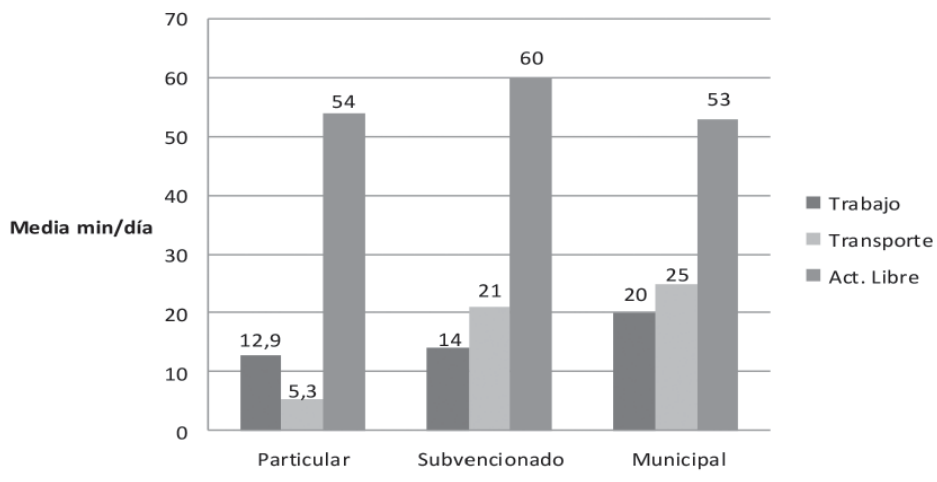

TABLA 1.

Nivel de actividad física, género y riesgo cardiovascular.

\begin{tabular}{|c|c|c|c|c|c|c|c|c|c|}
\hline & & \multicolumn{2}{|c|}{ Alto } & \multicolumn{2}{|c|}{ Moderado } & \multicolumn{2}{|c|}{ Bajo } & \multicolumn{2}{|c|}{ Total } \\
\hline & & Número & Porcentaje & Número & Porcentaje & Número & Porcentaje & Numero & Porcentaje \\
\hline \multirow[t]{3}{*}{ Género } & Femenino & 60 & $41,96 \%$ & 101 & $62,73 \%$ & 14 & $77,78 \%$ & 175 & $54,35 \%$ \\
\hline & Masculino & 83 & $58,04 \%$ & 60 & $37,27 \%$ & 4 & $22,22 \%$ & 147 & $45,65 \%$ \\
\hline & Total & $\begin{array}{l}143 \\
(44,4 \%)\end{array}$ & $100 \%$ & $\begin{array}{l}161 \\
(50 \%)\end{array}$ & $100 \%$ & $\begin{array}{l}18 \\
(5,6 \%)\end{array}$ & $100 \%$ & 322 & $100 \%$ \\
\hline Riesgo & Sin Riesgo & 117 & $81,82 \%$ & 134 & $82,23 \%$ & 14 & $77,78 \%$ & 265 & $82,30 \%$ \\
\hline \multirow[t]{2}{*}{ Cardiovascular } & $\begin{array}{l}\text { Con } \\
\text { Riesgo }\end{array}$ & 26 & $18,18 \%$ & 27 & $16,77 \%$ & 4 & $22,22 \%$ & 57 & $17,70 \%$ \\
\hline & Total & 143 & $100 \%$ & 161 & $100 \%$ & 18 & $100 \%$ & 322 & $100 \%$ \\
\hline
\end{tabular}


OMS (16), 62,73\% cumplía las recomendaciones mundiales de actividad física versus $37,26 \%$ que no se adhería (tabla 2 ). Se calculó la media de actividad física, siendo de 110,3 min/día en hombres, y 79,5 min/día en mujeres, existiendo una diferencia significativa entre ambos sexos $(p=0,002)$. En cuanto al cálculo del riesgo de no alcanzar las recomendaciones de la OMS, resultó ser superior en las mujeres (OR: 1,9; 95\%IC: 1,16-3,11).

El grado de adherencia a las recomendaciones de la OMS, según el tipo de establecimiento se muestra en la tabla 3. El cálculo de la media minutos/día de AF por establecimiento evidenció 97,1 min/día de AF para los municipalizados, 72 min/día de AF en particulares, y 94,6 min/día de AF en subvencionados, existiendo una diferencia significativa $(p=0,01)$.

Para los ámbitos de actividad física que considera el cuestionario GPAQ, y al calcular la media min/día del total de la muestra, se obtuvo un valor de 56,3 min/día para el ámbito "actividad libre"; 20,9 min/día en "transporte"; y 16,4 min/día en "trabajo" (colegio). En el gráfico 3 se muestra la media min/ día de AF en los tres tipos de establecimientos según ámbitos.

Respecto a lo anterior (gráfico 3), se demostró una correlación entre las variables $(p=0,019)$, también existieron diferencias significativas entre los ámbitos "actividad libre", $p=0,027$, en "transporte", $p=0,000$ y "trabajo", $p=0,000$ con el tipo de establecimiento.

La media minutos/día de comportamiento sedentario para el sexo masculino fue 669,5 minutos/día, y para las mujeres 663,5 minutos/día. No existiendo diferencia significativa $(p=0,654)$.

Según tipo de establecimiento educacional, la media minutos/día de comportamiento sedentario para establecimientos municipales fue $691,6 \mathrm{~min} /$ día; particulares 625,9 $\mathrm{min} /$ día y subvencionados $650,8 \mathrm{~min} /$ día, existiendo una diferencia significativa $(p=0,0001)$.

\section{DISCUSIÓN}

En el presente estudio se observó que 48,14\% de los adolescentes presentaba malnutrición por exceso. Destacando que existía una mayor prevalencia de obesidad en los establecimientos municipalizados, presentando $29,45 \%$ en comparación con los otros establecimientos, situación que pudiese atribuirse a factores socioeconómicos, y que coincide con lo publicado por Burrows y cols. quienes evidenciaron diferencias entre el IMC y los diferentes tipos de establecimientos educacionales (19).

Se observó que 17,7\% del total de la muestra presentó riesgo cardiovascular, cifras cercanas a lo informado por la prueba SIMCE el año 2012 para estudiantes de $8^{\circ}$ básico en la Región de Magallanes (6). El encontrar un bajo porcentaje de riesgo cardiovascular basado en el perímetro de cintura en esta muestra de adolescentes, en contraste con las elevadas cifras de malnutrición por exceso, pudiera explicarse debido a que el punto de corte para dicho parámetro propuesto en las tablas de referencia en este grupo etario es muy amplio, lo que pudiese condicionar que la mayoría de los estudiantes clasifiquen sin riesgo cardiovascular (1).

Las pruebas estadísticas no demostraron asociación entre riesgo cardiovascular y nivel de actividad física, pues quienes presentaban este factor de riesgo, demostraron realizar en su mayoría AF de intensidad alto-moderado, atribuible lo anterior, probablemente a la etapa de cambio en la que se encuentran los adolescentes evaluados, donde cobra relevancia factores tales como, el auto-concepto físico, el modelo estético y la imagen corporal, los cuales se asocian a la presión sociocultural en este grupo (20), lo que pudiera motivar el aumento de los niveles de actividad física a esta edad; sumado a que este factor de riesgo puede venir configurándose tempranamente desde la infancia, o incluso antes (21).

TABLA 2

Grado de adherencia a la recomendación de actividad física y género

\begin{tabular}{|c|c|c|c|c|c|}
\hline & & \multicolumn{2}{|c|}{ Cumple recomendaciones } & \multicolumn{2}{|c|}{ No cumple recomendaciones } \\
\hline & & Número & Porcentaje & Número & Porcentaje \\
\hline \multirow[t]{3}{*}{ Género } & Femenino & 98 & $48,51 \%$ & 77 & $64,17 \%$ \\
\hline & Masculino & 104 & $51,49 \%$ & 43 & $35,83 \%$ \\
\hline & Total & $202(62,73 \%)$ & $100 \%$ & $120(37,26 \%)$ & $100 \%$ \\
\hline
\end{tabular}

TABLA 3

Grado de adherencia a la recomendación de actividad física y tipo de establecimiento educacional.

\begin{tabular}{|c|c|c|c|c|c|}
\hline & & \multicolumn{2}{|c|}{ Cumple recomendaciones } & \multicolumn{2}{|c|}{ No Cumple recomendaciones } \\
\hline & & Numero & Porcentaje & Numero & Porcentaje \\
\hline \multirow[t]{4}{*}{ Establecimiento } & Particular & 17 & $8,42 \%$ & 14 & $11,67 \%$ \\
\hline & Subvencionado & 92 & $45,54 \%$ & 53 & $44,17 \%$ \\
\hline & Municipal & 93 & $46,04 \%$ & 53 & $44,17 \%$ \\
\hline & Total & 202 & $100 \%$ & 120 & $100 \%$ \\
\hline
\end{tabular}


La media minutos/día de AF por establecimiento evidenció una diferencia significativamente mayor en los establecimientos municipales, versus subvencionados y particulares. Habitualmente, se piensa que las personas con malnutrición por exceso, que en este estudio predominó en los establecimientos educacionales municipalizados, harían insuficiente o nula actividad física, sin embargo, los resultados obtenidos sugieren que el estado nutricional, no sería una condicionante para ello. Similares hallazgos fueron encontrados por Trejo y cols en una muestra de escolares mexicanos de 6 a 11 años de edad (22).

Respecto al grado de adherencia a las recomendaciones de AF de la OMS, resultó positivo observar que más de la mitad de la muestra $(62,73 \%)$, clasificó como "suficientemente activos", contrariamente a los resultados obtenidos de la ficha CLAP del año 2011 (4), en la cual, más de la mitad de los evaluados eran sedentarios (4,3 horas/semana de AF), en comparación a 10 horas/semana aproximadamente encontrados en el presente estudio.

La mayor adherencia a las recomendaciones de AF de la OMS encontrada para el sexo masculino, así como el tipo de AF que realizan, concuerda con otros estudios que diferencian a ambos géneros (23-25).

Considerando los 3 ámbitos donde se desarrollan las actividades físicas, $100 \%$ de los estudiantes realizaba AF de intensidad moderada en el trabajo (colegio), las cuales corresponden a la clase de educación física obligatoria con una duración de 90 minutos por semana en los tres tipos de establecimientos educacionales. Además, se evidenció en este estudio, que el tiempo efectivo de actividad física en el colegio es mucho menor, ya que se destina un tiempo para el traslado, cambio de vestuario, duchas e instrucciones de la clase.

Existe una diferencia notoria en cuanto a los minutos dedicados a la AF en los ámbitos de tiempo libre y desplazamientos, donde predomina el sexo masculino. En esta etapa, las mujeres adolescentes son menos activas que los hombres, lo cual podría asociarse al mayor porcentaje de obesidad en ellas en la etapa adulta (26).

La mayor contribución a la actividad física global en minutos/día, está dada en los ámbitos de transporte y tiempo libre, visualizándose que los estudiantes realizan diferentes deportes o actividades de intensidad vigorosa y/o moderada, después de sus actividades escolares o durante fines de semana.

En relación a los minutos/día de los tres ámbitos de actividad física, porcentualmente la "actividad libre" supera en más del doble a las actividades del "transporte". El bajo aporte de actividad física durante el transporte (desplazamientos) en relación a los otros dos ámbitos, pudiese deberse en la comuna de Punta Arenas a las cortas distancias, a la proporción vehículos/familia, disponibilidad de transporte público, factores climáticos, entre otros. No obstante, se observaron diferencias significativas por tipo de establecimiento en el ámbito del transporte, sobresaliendo los establecimientos municipalizados con mayor media min/día, lo que se puede atribuir a factores socioeconómicos.

Los hombres desarrollaban AF de mayor intensidad en los ámbitos de trasporte y tiempo libre que las mujeres, concordando estas diferencias por género según lo publicado en otros estudios, y atribuible probablemente a factores socioculturales (23).

La mayor cantidad de minutos/día de comportamiento sedentario observada en los establecimientos municipales, sobre los particulares y subvencionados, podría asociarse a factores socioeconómicos y culturales, donde pertenecer a estratos socioeconómicos bajos, ofrece menos alternativas y espacios para desarrollar la actividad física (27).

En base los hallazgos del presente estudio, Ilama la atención que los establecimientos municipales presentaban por una parte, un mayor comportamiento sedentario, y por otra, una media min/día de actividad física mayor. Estos resultados, plantean la necesidad de evaluar la consistencia del instrumento utilizado, ya que se consultan los minutos de sedentarismo, y no se consideran las horas destinadas de sueño, que al contrastarlas posteriormente con los minutos de AF diarios, pudiera ser la causa de esta dualidad.

Igualmente, se requiere contar con nuevas definiciones de sedentarismo. Pues se observan adolescentes "suficientemente activos", según criterios OMS, pero con altos niveles de sedentarismo, esto coincide con lo analizado por Carlos Cristi-Montero y Fernando Rodríguez (28).

\section{CONCLUSIÓN}

En este estudio, se observó que un alto porcentaje de adolescentes presentaba malnutrición por exceso, existiendo una mayor prevalencia de obesidad en los establecimientos municipalizados de la comuna de Punta Arenas.

Al analizar el riesgo cardiovascular basándose en el perímetro de cintura, se observó, que los puntos de corte para este grupo son muy amplios, razón que pudiera explicar el bajo riesgo cardiovascular al utilizar este parámetro (17,7\%).

Los estudiantes de 14 y 15 años de la ciudad de Punta Arenas, realizan actividad física a diversas intensidades, siendo esta mayor durante su tiempo libre.

Existían diferencias entre los distintos tipos de establecimientos educacionales, y los tres ámbitos encuestados donde se realiza la actividad física, con una mayor significancia en el ámbito del transporte (desplazamientos), predominando los establecimientos municipalizados, asociado lo anterior, probablemente a factores socioeconómicos.

La media minutos/día de AF por tipo de establecimiento es mayor en los municipalizados.

Se demostró una diferencia significativa de la media minutos/día de AF entre ambos sexos, siendo mayor en el sexo masculino.

La mayoría adhiere a las recomendaciones establecidas por la OMS. Observándose mayor riesgo de no cumplir estas recomendaciones en el sexo femenino.

El encontrar por un lado a la mayoría de los adolescentes en la categoría suficientemente activos según la clasificación OMS, pero con altos niveles de sedentarismo, lleva a la necesidad de revisar estos conceptos, así como, las recomendaciones de actividad física para este grupo, y talvez complementar este cuestionario con mediciones más objetivas .

La importancia de este estudio radica en que la información recopilada contribuirá a caracterizar aún más al grupo adolescente, aportando mayores antecedentes junto a otros análisis hechos a nivel país y Región de Magallanes, con datos acerca del nivel de actividad física y en los distintos ámbitos de participación, y considerando que el efecto multiorgánico beneficioso de la AF y del ejercicio, tienen una clara dependencia dosis/respuesta, la que depende tanto de su duración, como de la intensidad, complementando así, estudios que evalúan otros aspectos de la condición física (condición física estructural y funcional).

\section{RECOMENDACIONES}

Debido a que los estudiantes pasan la mayor parte del día en su establecimiento educacional, siendo esta una actividad predominantemente sedentaria, es que se refuerza la 
necesidad de realizar intervenciones que consideren el tipo, frecuencia y duración efectiva de la actividad física. También, se sugiere incorporar otro tipo de actividades tales como, pausas activas, cambio de salas durante el horario de clases, a modo de disminuir los tiempos de inactividad durante la extensa jornada escolar.

Finalmente, se considera que es importante seguir promoviendo el aumento de los niveles de intensidad de la actividad física en la población, para ello se debe insistir en incluir en las estrategias de salud recomendaciones sobre disminuir las horas frente al televisor o estar acostado, fomentar interrupciones al hábito sedentario, así como, incrementar el tiempo durante el día destinado a actividades de intensidad ligera, las que igualmente producirían beneficios en la salud, contribuyendo a disminuir las enfermedades crónicas no transmisibles.

\section{RESUMEN}

Objetivo: El objetivo de este estudio fue identificar el grado de adherencia a las recomendaciones mundiales sobre actividad física, así como su asociación con riesgo cardiovascular, variables antropométricas y socio-demográficas en adolescentes de la comuna de Punta Arenas. Sujetos y métodos: Se obtuvo una muestra aleatoria, estratificada y proporcional de 322 estudiantes de establecimientos particular-pagados, particular-subvencionados y municipalizados. Se aplicó el cuestionario mundial sobre actividad física (GPAQv2), y mediciones antropométricas de peso, talla, perímetro de cintura, y clasificación del estado nutricional según normas MINSAL. Resultados: El 50,31\% del total de la muestra tenía un estado nutricional normal y $48,14 \%$ presentó malnutrición por exceso. También, 666,8 min/día de sedentarismo promedio entre las medias por sexo. El sexo femenino presenta un OR: 1,9; 95\%IC: 1,16-3,11 según recomendaciones de la OMS. Discusión: Se encontró a la mayoría de los estudiantes evaluados en la categoría "suficientemente activos" según las recomendaciones de la OMS. Se observó un alto porcentaje sin riesgo cardiovascular (82,30\%). Conclusión: El nivel de actividad física no estaría condicionado por el estado nutricional, evidenciándose en la mayoría de los alumnos la realización de actividad física moderada en su tiempo libre.

Palabras clave: Actividad física; GPAQ; riesgo cardiovascular; adolescentes; estado nutricional.

\section{BIBLIOGRAFÍA}

1. Ministerio de Salud de Chile. Orientaciones Técnicas para el control de salud integral de los adolescentes. Control Joven Sano 2014. Disponible en: http://web.minsal.cl/ sites/default/files/files/2014controlsaludadolescente.pdf. Citado el 01/09/2014.

2. Ministerio de Salud de Chile. Estrategia Nacional de Salud para el cumplimiento de los objetivos sanitarios de la década 2011-2020. Disponible en: http://web.minsal.cl/ portal/url/item/c4034eddbc96ca6de0400101640159b8. pdf. Citado el 01/09/2014.

3. Ministerio de Salud de Chile. Encuesta Nacional de Salud ENS 2009-2010. Disponible en: http://web.minsal.cl/ portal/url/item/bcb03d7bc28b64dfe040010165012d23. pdf. Citado el 01/09/2014.

4. Ministerio de Salud de Chile. Programa Nacional de Salud Integral de adolescentes y jóvenes. Julio 2012. Disponible en:

http://web.minsal/cl/sites/default/files/estudiosituacionadolescente.pdf. Citado el 01/09/2014.

5. Ministerio de Salud de Chile. Gobierno de Chile. Encuesta mundial de Salud Escolar Chile 2004. Disponible en: http://www.who.int/chp/gshs/Chile_questionnaire_spanish_2004.pdf

6. Gobierno de Chile. Agencia de la calidad de la Educación. SIMCE Educación Física 2012. Disponible en:

http://www.agenciaeducación.cl/wp-content/files_mf/ ir_educación_fisica_2012.pdf.

7. Gobierno de Chile. Junta Nacional de Auxilio Escolar y Becas. Informe Mapa Nutricional 2013. Disponible en: http://www.junaeb.cl/wp-content/uploads/2013/03/ Informe-Mapa-Nutricional-2013.pdf.

8. Katzmarzyk PT, Srinivasan SR, Chen W, Malina RM, Bouchard C, Berenson GS. Body mass index, waist circumference, and clustering of cardiovascular disease risk factors in a biracial sample of children and adolescents. Pediatrics 2004; 114: e198-205.

9. Caballero AE, Bousquet-Santos K, Robles-Osorio L, Montagnani V, Soodini G, Porramatikul S, et al. Overweight Latino children and adolescents have marked endothelial dysfunction and subclinical vascular inflammation in association with excess body fat and insulin resistance. Diabetes Care 2008; 31: 576-82.

10. Cook $S$, Weitzman $M$, Auinger $P$, Nguyen $M$, Dietz WH. Prevalence of a metabolic syndrome phenotype in adolescents: Findings from the third national health and nutrition examination survey, 1988-1994. Arch Pediatr Adolesc Med. 2003; 157: 821-7.

11. Weiss R, Dziura J, Burgert TS, Tamborlane WV, Taksali E, Yeckel CW, et al. Obesity and the metabolic syndrome in children and adolescents. N Engl J Med. 2004; 350: 2362-74.

12. Barja S, Arteaga A, Acosta AM, Hodgson MI. [Insulin resistance and other expressions of metabolic syndrome in obese Chilean children]. Rev Med Chil. 2003; 131: 259-68.

13. Burrows $R$, Burgueno $M$, Leiva $L$, Ceballos $X$, Guillier I, Gattas $V$, et al. [Cardiovascular risk and metabolic profile in obese children and adolescents with low insulin sensitivity]. Rev Med Chil. 2005; 133: 795-804.

14. Armstrong T, Bull F.Development of the World Health Organization Globay Physical Activity Questionnaire. JPublic Health.2006; 14:66-70. www.who.int/chp/steps/ resources/GPAQ_Analysis_Guide.pdf.

15. http://www.uchile.cl/portal/investigacion/centrointerdisciplinario-de-estudios-en bioetica/documentos/75657/documentos-de-consentimiento-informadoelaborados-por-la.oms.

16. Organización Mundial de la Salud (OMS). Recomendaciones mundiales sobre actividad física para la salud: ISBN: 9789241599979. Año, 2010

17. Arnaiz $P$, Grob F, Cavada $G$, Dominguez A, Bancalari $R$, Cerda B,Zamorano J; Fernández M, García H. Razón cintura estatura en escolares no varía con el género, la edad ni la maduración puberal. Rev Med Chil. 2014; 142: 574-8.

18. Pangrazi RP, Beighle $A$, Vehige $T$, Vack $C$. Impact of promoting lifestyle activity for youth (PLAY) on children's physical activity. J Sch Health 2003; 73 (8): 317-21.

19. Burrows $A$, Raquel et al. Hábitos de ingesta y actividad física en escolares, según tipo de establecimiento al que asisten. Rev. méd. Chile [online]. 2008, vol.136, n.1 [citado 2015-08-09], pp. 53-63. Disponible en: http://www.scielo.cl/scielo.php?script=sci_ arttex\&pid=S0034-9887200800010007\&Ing=es\&nrm= iso. ISSN 0034-9887. 
20. Fernández $M$, Juan J, Arbonés $M$, Blanco $M$. Autoconcepto físico, modelo estético e imagen corporal en una muestra de adolescents. ISSN 0210-8348, 1999, 20 (1), p 27-38.

21. Ramírez R. Programación fetal in útero y su impacto en la salud del adulto. Endocrinol Nutr. 2012;59(6):383-93.

22. Ortiz P, Chairez S, Mollinedo E, Lugo G. Relación entre actividad física y obesidad en escolares. Rev Cubana Med General Integral. 2012; 28(1):34-41.

23. Abarca S, Zaragoza J, Generelo E, Clemente J. Comportamientos sedentarios y patrones de actividad física en adolescents. Rev. Int. Med. Cienc. Act. Fis. Deporte. Vol. 10 - número 39 - septiembre 2010 - ISSN: 1577 - 0354.

24. Castillo I, Balaguer I. Patrones de actividades físicas en niños y adolescents. Rev Educ Física Deportes. 199; 8 (54): 22-29.
25. Gonzalez G, Llanos J. Adolescencia, actividad física y factores metabólicos de riesgo cardiovascular. Rev Esp Cardiol. 2007; 60:565-8.

26. Chillon P, Tercedor P, Delgado M, González M. Actividad físico-deportiva en escolares adolescents. Retos. Nuevas Tendencias Educ Física, Deporte Recreación 2002; 1, 5-12.

27. Babey $\mathrm{SH}$, Wolstein J, Diamant AL. Role models and social supports related to adolescent physical activity and overweight/obesity. Policy Brief UCLA Cent Health Policy Res. 2015; (PB2015-3):1-8.

28. Cristi-Montero, Carlos, \& Rodríguez, R, Fernando. (2014). Paradoja "activo físicamente pero sedentario, sedentario pero activo físicamente": Nuevos antecedentes, implicaciones en la salud y recomendaciones. Rev Méd Chil. $142(1), 72-8$. 